\title{
Scanning Electron Microscope Point Spread Function Determination Through the Use of Particle Dispersions
}

\author{
Matthew D. Zotta ${ }^{1,2}$ and Eric Lifshin ${ }^{1}$ \\ ${ }^{1 .}$ Colleges of Nanoscale Science and Engineering, SUNY Polytechnic Institute, Albany, NY, USA \\ 2. Nanojehm Inc., Albany, NY, USA
}

Over the years, significant advances have been made in scanning electron microscope (SEM) resolution primarily due to hardware improvements including higher brightness electron sources with smaller energy spreads and better electron optics that decrease the final probe size. Although these advancements represent significant developments, they commonly come with added complexity and cost. In addition, while a small probe diameter may be necessary for high resolution imaging, it may not be attainable in all instances, most notably low voltage where chromatic aberrations in the electron optics limit the minimum probe size. Other scenarios such as long working distance settings as with retractable backscatter detectors and other analytical operating conditions can lead to larger beams at the sample surface. Situations in which there is a need for a high signal to noise ratio can also present an issue as the probe size often increases with probe current leading to decreased resolution due to beam broadening.

Given the above considerations, the research presented here has focused on determining the crosssectional distribution of the electrons in the beam known as the point spread function (PSF). When the electron beam lands on a sample area centered on a given pixel, signal electrons (secondary, backscatter, transmitted) are collected from the entire surface area that the beam covers. In addition, it is well known that depending on the landing energy of the electrons and thickness of the sample, a tear-drop shaped interaction volume of several times the initial impact area can be a source of signal electrons as well. Assuming this interaction volume is small, as in the case of a very thin sample, the primary cause of blurring in an image will be the result of attempting to measure detail smaller than the diameter of the beam. For thermionic SEMs this can occur at pixel sizes $\lesssim 3 \mathrm{~nm}$ and for Schottky at pixel sizes $\lesssim 1 \mathrm{~nm}$. The technique employed here utilizes a novel hardware/software package that exploits the high signal to noise ratio of gold nanoparticles and the small interaction volume of ultra-thin carbon coated TEM grids to determine the distribution of electrons in an electron beam. Techniques exist for measuring the SEM PSF which include taking an image of a standard where the standard had been previously measured with a high resolution SEM. [1,2,3] By comparing the observed image with the high resolution image, one can determine the PSF. This process is, however, time consuming and impractical for routine use. The method discussed here represents a significant advance as the PSF can now be determined without the need for a standard previously measured by a high resolution instrument.

The user inserts a calibration standard into the SEM and images it (Fig. 1a) at whatever conditions desired for imaging; this includes accelerating voltage, working distance, astigmatism, probe current and anything else that might affect the shape and distribution of the electron beam. As mentioned above, the calibration standard consists of a distribution of gold nanoparticles having a known shape and size dispersed on an ultra-thin carbon coated TEM grid. The user loads the calibration image into the Nanojehm Aura workstation which has been developed to efficiently process the image and compute the PSF. The software auto-locates particles in the field that fit within the size and shape tolerances expected for the sample particles and crops them out individually. The software combines the individual 
cropped particles into a single composite particle having a high signal to noise ratio as shown by Fig. $1 \mathrm{~b}$. An ultra-high resolution reference image of a particle is theoretically generated having the specified size, shape, composition, and pixel size corresponding to the particles found in the field as shown in Fig 1c. The workstation is able to take the reference image and the composite particle image and determine the SEM PSF for the operating conditions specified as shown in Fig. 2. The PSF can then be used in conjunction with deconvolution technique to restored additional images taken under the same imaging conditions. $[2,3,4,5]$

\section{References:}

[1] Kandel, Y.P. et al, Microscopy and Microanalysis 21(S3) (2015) p. 699.

[2] Lifshin, E., Kandel, Y.P. and Moore, R.L., Microscopy and Microanalysis, 20(1) (2014), p. 78.

[3] Lifshin, E. et al, Microscopy and Microanalysis 20(S3) (2014) p. 12.

[4] Lifshin, E. et al, Microscopy and Microanalysis 20(S3) (2014) p. 386.

[5] Zotta, M.D. et al, Microscopy and Microanalysis 21(S3) (2015) p. 33.
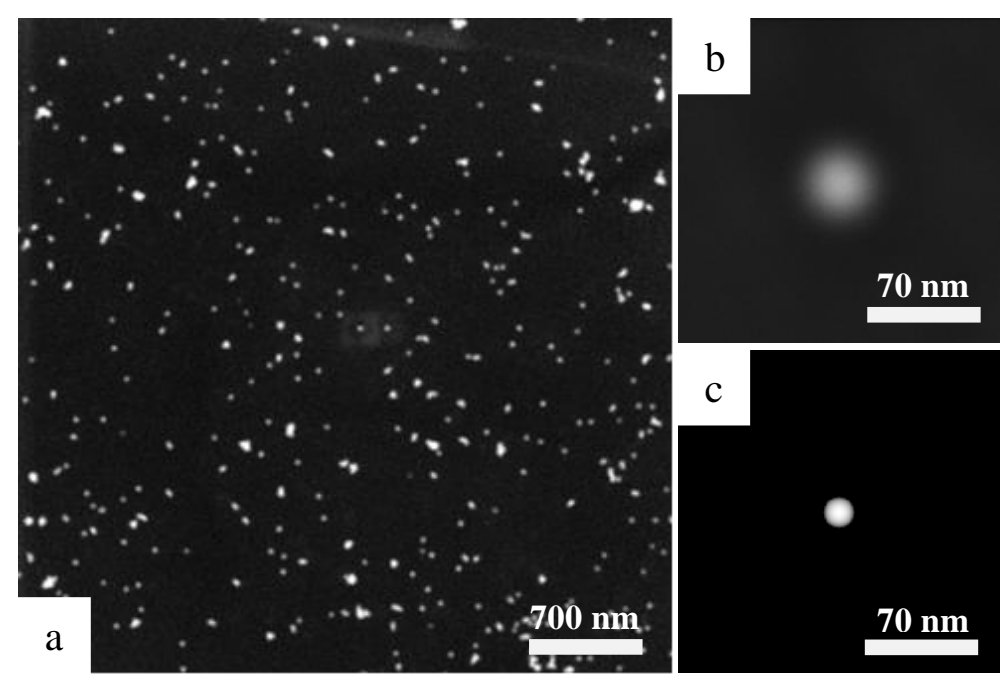

Figure 1. (a) The calibration image taken with a TESCAN VEGA ${ }^{\circledR} \mathrm{LaB}_{6} \mathrm{SEM}, \mathrm{BI}=3, \mathrm{HV}=20 \mathrm{keV}$, (b) the stacked particle image created from selected particles in the calibration image and (c) the theoretically generated high resolution reference image for the given operating conditions.
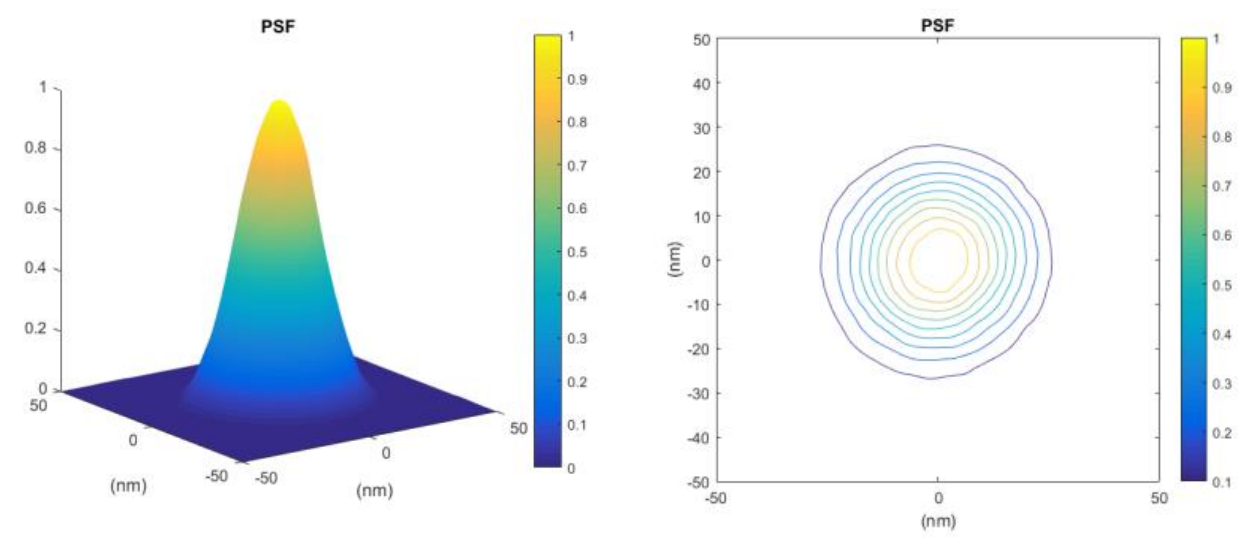

Figure 2. The calculated PSF from the image in Fig.1a and Fig. 1b shown in a (left) surface view and (right) contour plot. 